\title{
Graph-based classification of multiple observation sets
}

\author{
E. Kokiopoulou ${ }^{\mathrm{a}, *}$, P. Frossard ${ }^{\mathrm{b}}$ \\ a Seminar for Applied Mathematics, ETH, Zurich, CH-8092, Switzerland \\ b Signal Processing Laboratory (LTS4), Ecole Polytechnique Fédérale de Lausanne (EPFL), Lausanne, CH-1015, Switzerland
}

\section{A R T I C L E I N F O}

\section{Article history:}

Received 27 November 2009

Received in revised form

26 May 2010

Accepted 9 July 2010

\section{Keywords:}

Graph-based classification

Multiple observations sets

Video face recognition

Multi-view object recognition

\begin{abstract}
A B S T R A C T
We consider the problem of classification of an object given multiple observations that possibly include different transformations. The possible transformations of the object generally span a low-dimensional manifold in the original signal space. We propose to take advantage of this manifold structure for the effective classification of the object represented by the observation set. In particular, we design a low complexity solution that is able to exploit the properties of the data manifolds with a graph-based algorithm. Hence, we formulate the computation of the unknown label matrix as a smoothing process on the manifold under the constraint that all observations represent an object of one single class. It results into a discrete optimization problem, which can be solved by an efficient and simple, yet effective, algorithm. We demonstrate the performance of the proposed graph-based algorithm in the classification of sets of multiple images. Moreover, we show its high potential in video-based face recognition, where it outperforms state-of-the-art solutions that fall short of exploiting the manifold structure of the face image data sets.
\end{abstract}

(c) 2010 Elsevier Ltd. All rights reserved.

\section{Introduction}

Recent years have witnessed a dramatic growth of the amount of digital data that is produced by sensors or computers of all sorts. That creates the need for efficient processing and analysis algorithms in order to extract the relevant information contained in these data sets. In particular, it commonly happens that multiple observations of an object are captured at different time instants or under different geometric transformations. For instance, a moving object may be observed over a time interval by a surveillance camera (see Fig. 1(a)) or under different viewing angles by a network of vision sensors (see Fig. 1(b)). This typically produces a large volume of multimedia content that lends itself as a valuable source of information for effective knowledge discovery and content analysis. In this context, classification methods should be able to exploit the diversity of the multiple observations in order to provide increased classification accuracy [17].

We build on our previous work [8] and we focus here on the pattern classification problem with multiple observations. We further assume that observations are produced from the same object under different transformations, so that they all lie on the

\footnotetext{
This work has been mostly performed while the first author was with the Signal Processing Laboratory (LTS4) of EPFL. It has been partly supported by the Swiss National Science Foundation, under Grant NCCR IM2.

* Corresponding author.

E-mail addresses: effrosyni.kokiopoulou@sam.math.ethz.ch (E. Kokiopoulou), pascal.frossard@epfl.ch (P. Frossard).
}

same low-dimensional manifold. We propose a novel graph-based algorithm inspired by label propagation [22]. Label propagation methods typically assume that the data lie on a low dimensional manifold living in a high dimensional space. They rely upon the smoothness assumption, which states that if two data samples $x_{1}$ and $x_{2}$ are close, then their labels $y_{1}$ and $y_{2}$ should be close as well. The main idea of these methods is to build a graph that captures the geometry of this manifold as well as the proximity of the data samples. The labels of the test examples are derived by "propagating" the labels of the labelled data along the manifold, while making use of the smoothness property. We exploit the specificity of our particular classification problem and constrain the unknown labels to correspond to one single class. This leads to the formulation of a discrete optimization problem that can be optimally solved by a simple and low complexity algorithm.

We apply the proposed algorithm to the classification of sets of multiple images in handwritten digit recognition, multi-view object recognition or video-based face recognition. In particular, we show the high potential of our graph-based method for efficient classification of images that belong to the same data manifold. For example, the proposed solution, despite its simplicity, outperforms state-of-the-art subspace or statistical classification methods in video-based face recognition and object recognition from multiple image sets. Hence, this paper brings new insight from the graph-based algorithms into the problems of multi-view object recognition or video-based face recognition, which - to the best of our knowledge-has not been offered by any of the existing approaches that are mainly categorized as either statistical or subspace ones. 
a

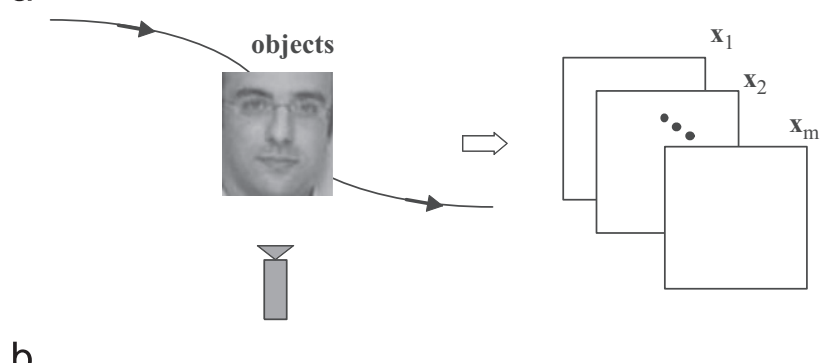

b

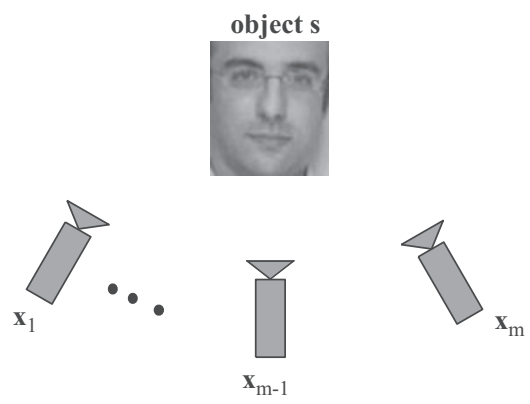

Fig. 1. A typical scenarios of producing multiple observations of an object. (a) Video frames of a moving object and (b) network of vision sensors.

The paper is organized as follows. We first formulate the problem of classification of multiple observation sets in Section 2. We introduce our graph-based algorithm inspired by label propagation in Section 3. Then we demonstrate the performance of the proposed classification method for handwritten digit recognition, object recognition and video-based face recognition in Sections 4.1, 4.2 and 5, respectively.

\section{Problem definition}

We address the problem of the classification of multiple observations of the same object, possibly with some transformations. In particular, the problem is to assign multiple observations of the test pattern/object $s$ to a single class of objects. We assume that we have $m$ multiple observations of $s$ of the following form:

$x_{i}^{(u)}=o_{i}(s), \quad i=1, \ldots, m$.

In the case of visual objects for example, $o_{i}(s)$ may correspond to a rotation, scaling, translation, or perspective projection of the object $s$, or $o_{i}(s)$ may correspond to an observation of a moving object $s$ obtained at a certain time step $t_{i}$. The superscript index $(u)$ in (1) simply denotes that the different observations are unlabelled. We assume that each observation $x_{i}^{(u)}$ is distinct from its peers (i.e., $x_{i}^{(u)} \neq x_{j}^{(u)}$, for $i \neq j$ ). Notice the common dependence of the $x_{i}^{(u)}$ on $s$, which further implies that the unknown class label of all $x_{i}^{(u)}$ is the same as that of $s$. The problem then is to classify $s$ in one of the $c$ classes under consideration, using the multiple observations $x_{i}^{(u)}, i=1, \ldots, m$.

In order to address the classification problem, we assume that we also have a training data set in our disposal. Therefore, one can organize the whole data set in two parts $X=\left\{X^{(l)}, X^{(u)}\right\}$, where $X^{(l)}=\left\{x_{1}, x_{2}, \ldots, x_{l}\right\} \subset \mathbb{R}^{d}$ and $X^{(u)}=\left\{x_{l+1}, \ldots, x_{n}\right\} \subset \mathbb{R}^{d}$, where $n=$ $l+m$. Let also $\mathcal{L}=\{1, \ldots, c\}$ denote the label set. The $l$ examples in $X^{(l)}$ are labelled $\left\{y_{1}, y_{2}, \ldots, y_{l}\right\}, y_{i} \in \mathcal{L}$, and the $m$ examples in $X^{(u)}$ are unlabelled. We associate the set of unlabelled data $X^{(u)}$ with the set of multiple observations introduced in (1), i.e., $X^{(u)}=\left\{x_{l+1}, \ldots, x_{n}\right\} \triangleq\left\{x_{1}^{(u)}, \ldots, x_{m}^{(u)}\right\}$. The classification problem can be formally defined as follows.
Problem 1. Given a set of labelled data $X^{(l)}$, and a set of unlabelled data $X^{(u)} \triangleq\left\{x_{j}^{(u)}=o_{j}(s), j=1, \ldots, m\right\}$ that correspond to multiple observations of $s$, the problem is to predict the correct class $c^{*}$ of the original pattern $s$.

One may view Problem 1 as a special case of semi-supervised learning [3], where the unlabelled data $X^{(u)}$ represent the multiple observations with the extra constraint that all unlabelled data examples belong to the same (unknown) class. The problem then resides in estimating the single unknown class, while generic semi-supervised learning problems attribute the test examples to different classes. We propose in the next section a novel efficient algorithm inspired from label propagation in order to solve Problem 1.

\section{Graph-based classification}

\subsection{Label propagation}

We review quickly here the classical label propagation algorithm and we later present our solution to Problem 1. The label propagation algorithm [22] is based on a smoothness assumption, which intuitively states that if $x_{1}$ and $x_{2}$ are closeby, then there is a high chance that they share the same class label. Denote by $\mathcal{M}$ the set of matrices of size $n \times c$ with nonnegative entries. Notice that any matrix $M \in \mathcal{M}$ provides a labelling of the data set by applying the following rule: $y_{i}=\max _{j=1, \ldots, c} M_{i j}$. We denote the initial label matrix as $Y \in \mathcal{M}$ where $Y_{i j}=1$ if $x_{i}$ belongs to class $j$ and 0 otherwise. The label propagation algorithm first forms the $k$ nearest neighbor $(k-\mathrm{NN})$ graph defined as

$\mathcal{G}=(\mathcal{V}, \mathcal{E})$,

where the vertices $\mathcal{V}$ correspond to the data samples $X$. An edge $e_{i j} \in \mathcal{E}$ is drawn if and only if $x_{j}$ is among the $k$ nearest neighbors of $x_{i}$.

It is common practice to assign weights on the edge set of $\mathcal{G}$. One typical choice is the Gaussian weights

$H_{i j}=\left\{\begin{array}{lr}\exp \left(-\frac{\left\|x_{i}-x_{j}\right\|^{2}}{2 \sigma^{2}}\right) & \text { when }(i, j) \in \mathcal{E}, \\ 0 & \text { otherwise. }\end{array}\right.$

The normalized Laplacian matrix $S \in R^{n \times n}$ is then defined as

$S=D^{-1 / 2} H D^{-1 / 2}$,

where $D$ is a diagonal matrix with entries $D_{i i}=\sum_{j=1}^{n} H_{i j}$. The matrix $S$ is also known as the similarity matrix (see, e.g., [2]), since the $(i, j)$ entry captures the similarity between $x_{i}$ and $x_{j}$. See also Fig. 2 for a schematic illustration of the $k$-NN graph and related notation.

Next, the algorithm computes a real valued $M^{*} \in \mathcal{M}$ based on which the final classification is performed using the rule $y_{i}=\max _{j=1, \ldots, c} M_{i j}^{*}$. This is done via a regularization framework with a cost function defined as

$\mathcal{U}(M)=\frac{1}{2}\left(\sum_{i, j=1}^{n} H_{i j}\left\|\frac{1}{\sqrt{D_{i i}}} M_{i}-\frac{1}{\sqrt{D_{j j}}} M_{j}\right\|^{2}+\mu \sum_{i=1}^{n}\left\|M_{i}-Y_{i}\right\|^{2}\right)$,

where $M_{i}$ denotes the $i$ th row of $M$. The computation of $M^{*}$ is done by solving the quadratic optimization problem $M^{*}=\operatorname{argmin}_{M \in \mathcal{M}} \mathcal{U}(M)$.

Intuitively, we are seeking an $M^{*}$ that is smooth along the edges of similar pairs $\left(x_{i}, x_{j}\right)$ and at the same time close to $Y$ when evaluated on the labelled data $X^{(l)}$. The first term in (4) is the smoothness term and the second is the fitness term.

Notice that when two examples $x_{i}$ and $x_{j}$ are similar (i.e., the weight $H_{i j}$ is large) minimizing the smoothness term in (4) results 


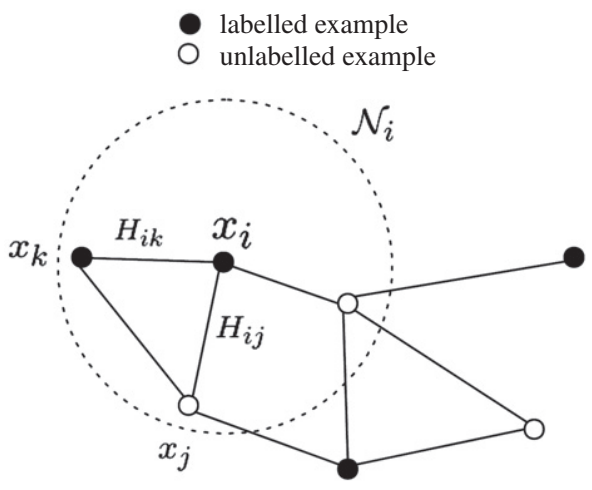

Fig. 2. A typical structure of the $k$-NN graph. $\mathcal{N}_{i}$ represents the neighborhood of the sample $x_{i}$.

in $M$ being smooth across similar examples. Thus, similar data examples will likely share the same class label. It can be shown [22] that the solution to problem (4) is given by

$M^{*}=\beta(I-\alpha S)^{-1} Y$,

where $\alpha=1 /(1+\mu)$ and $\beta=\mu /(1+\mu)$.

Finally, several other variants of label propagation have been proposed in the past few years. We mention for instance, the method of [25] and the variant of label propagation that was inspired from the Jacobi iteration algorithm [3, Ch. 11]. Finally, it is interesting to note that there have also been found connections to Markov random walks [18] and electric networks [26]. Note finally that label propagation is probably the most representative algorithm among the graph-based methods for semi-supervised learning.

\subsection{Label propagation with multiple observations}

We propose now to build on graph-based algorithms to solve the problem of classification of multiple observation sets. In general, the classical label propagation framework assumes that the unlabelled examples come from different classes. As Problem 1 presents the specific constraint that all unlabelled data belong to the same class, label propagation does not fit exactly the definition of the problem as it falls short of exploiting its special structure. Therefore, we propose in the sequel a novel graphbased algorithm inspired from label propagation, which (i) uses the smoothness criterion on the manifold in order to predict the unknown class labels and (ii) at the same time, it is able to exploit the specificity of Problem 1.

We represent the data labels with a 1-of-c encoding, which permits to form a binary label matrix of size $n \times c$, whose $i$ th row encodes the class label of the $i$ th example. The class label is basically encoded in the position of the nonzero element.

Suppose now that the correct class for the unlabelled data is the $p$ th one. In this case, we denote by $Z_{p} \in R^{n \times c}$ the corresponding label matrix. Note that there are $c$ such label matrices; one for each class hypothesis. Each class-conditional label matrix $Z_{p}$ has the following form:

$Z_{p}=\left[\begin{array}{c}\frac{Y_{l} \in R^{l \times c}}{1 e_{p}^{\top} \in R^{m \times c}}\end{array}\right] \in R^{n \times c}$,

where $e_{p} \in R^{c}$ is the $p$ th canonical basis vector and $\mathbf{1} \in R^{m}$ is the vector of ones. Fig. 3 shows schematically the structure of the matrix $Z_{p}$. The upper part corresponds to the labelled examples

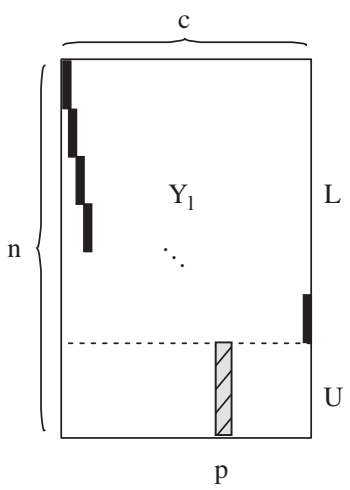

Fig. 3. Structure of the class-conditional label matrix $Z_{p}$.

and the lower part to the unlabelled ones. $Z_{p}$ holds the labels of all data samples, assuming that all unlabelled examples belong to the $p$ th class. Observe that the $Z_{p}$ 's share the first part $Y_{l}$ and differ only in the second part.

Since all unlabelled examples share the same label, the class labels have a special structure that reflects the special structure of Problem 1, as outlined in our previous work [8]. We could then express the unknown label matrix $M$ as,

$M=\sum_{p=1}^{c} \lambda_{p} Z_{p}, \quad Z_{p} \in R^{n \times c}$,

where $Z_{p}$ is given in (6), $\lambda_{p} \in\{0,1\}$ and

$\sum_{p=1}^{c} \lambda_{p}=1$

In the above, $\lambda=\left[\lambda_{1}, \ldots, \lambda_{c}\right]$ is the vector of linear combination weights, which are discrete and sum up to one. Ideally, $\lambda$ should be sparse with only one nonzero entry pointing to the correct class.

The classification problem now resides in estimating the proper value of $\lambda$. We rely on the smoothness assumption and we propose the following objective function:

$\tilde{\mathcal{Q}}(M(\lambda))=\frac{1}{2}\left(\sum_{i, j=1}^{n} H_{i j}\left\|\frac{1}{\sqrt{D_{i i}}} M_{i}-\frac{1}{\sqrt{D_{j j}}} M_{j}\right\|^{2}\right)$,

where the optimization variable now becomes the $\lambda$ vector. Notice that the fitting term in the classical label propagation algorithm (see Eq. (4)) is not needed anymore due to the structure of the $Z$ matrices. Furthermore, we observe that the optimization parameter $\lambda$ is implicitly represented in the above equation through $M$, defined in Eq. (7).

In the above, $M_{i}$ (resp. $M_{j}$ ) denotes the $i$ th (resp. $j$ th) row of $M$. In the case of normalized similarity matrix, the above criterion becomes

$\mathcal{Q}(M(\lambda))=\frac{1}{2} \sum_{i, j=1}^{n} S_{i j}\left\|M_{i}-M_{j}\right\|^{2}$,

where $S$ is defined as in (3). It can be seen that the objective function directly relies on the smoothness assumption. When two examples $x_{i}, x_{j}$ are nearby (i.e., $H_{i j}$ or $S_{i j}$ is large), minimizing $\tilde{Q}(\lambda)$ and $Q(\lambda)$ result in $M_{i}$ being close to $M_{j}$, which in turn results in similar class labels $y_{i}$ and $y_{j}$. Therefore, objects in the same class contribute to a low cost as long as their labels are identical. Objects that are very dissimilar have a low value $S_{i j}$, which gives a small importance on the value of their respective labels. This is 
exactly the goal of the smoothness constraint in our optimization problem.

The following proposition now shows the explicit dependence of $Q$ on $\lambda$.

Proposition 1. Assume the data set is split into $l$ labelled examples $X^{(l)}$ and $m$ unlabelled examples $X^{(u)}$, i.e., $X=\left[X^{(l)}, X^{(u)}\right]$. Then, the objective function (10) can be written in the following form:

$Q(\lambda)=C+\frac{1}{2} \sum_{i \leq l, j>l} S_{i j}\left\|Y_{i}-\lambda\right\|^{2}+\frac{1}{2} \sum_{i>l, j \leq l} S_{i j}\left\|Y_{j}-\lambda\right\|^{2}$,

where $C=\sum_{i \leq l, j \leq l} S_{i j}\left\|Y_{i}-Y_{j}\right\|^{2}$.

Proof. From Eq. (10) observe that

$$
\begin{aligned}
\mathcal{Q}(\lambda)= & \underbrace{\frac{1}{2} \sum_{i, j \leq l}^{n} S_{i j}\left\|M_{i}-M_{j}\right\|^{2}}_{Q_{1}}+\underbrace{\frac{1}{2} \sum_{i, j>l}^{n} S_{i j}\left\|M_{i}-M_{j}\right\|^{2}}_{Q_{2}} \\
& +\underbrace{\frac{1}{2} \sum_{i \leq l, j>l}^{n} S_{i j}\left\|M_{i}-M_{j}\right\|^{2}}_{Q_{3}}+\underbrace{\frac{1}{2} \sum_{i>l, j \leq l}^{n} S_{i j}\left\|M_{i}-M_{j}\right\|^{2}}_{Q_{4}} .
\end{aligned}
$$

We consider the following cases:

(i) $i \leq l$ and $j \leq l$ : both data examples $x_{i}$ and $x_{j}$ are labelled. Then, $M_{i}=\left(\sum_{p=1}^{c} \lambda_{p}\right) Y_{i}=Y_{i}$, due to the special structure of the $Z$ matrices (see (6)) and also due to the constraint from Eq. (8). Similarly, $M_{j}=Y_{j}$. This results in $Q_{1}=\frac{1}{2} \sum_{i, j \leq l}$ $S_{i j}\left\|Y_{i}-Y_{j}\right\|^{2}=C$, which is a constant term and does not depend on $\lambda$.

(ii) $i>l$ and $j>l$ : both data samples $x_{i}$ and $x_{j}$ are unlabelled. In this case, $M_{i}=\lambda$ and $M_{j}=\lambda$, again due to (6). Therefore the second term $Q_{2}$ is zero.

(iii) $i \leq l$ and $j>l$ : $x_{i}$ is labelled and $x_{j}$ is unlabelled. In this case, $M_{i}$ $=Y_{i}$ and $M_{j}=\lambda$. This results in $Q_{3}=\frac{1}{2} \sum_{i \leq l, j>l} S_{i j}\left\|Y_{i}-\lambda\right\|^{2}$.

(iv) $i>l$ and $j \leq l$ is analogous to the case (iii) above, where the roles of $x_{i}$ and $x_{j}$ are switched. Thus, $Q_{4}=\frac{1}{2} \sum_{i>l, j \leq l} S_{i j}\left\|Y_{j}-\lambda\right\|^{2}$.

Putting the above facts together yields Eq. (11).

The above proposition suggests that only the interface between labelled and unlabelled examples matters in determining the smoothness value of a candidate label matrix $M$, or equivalently the solution vector $\lambda$. We use this observation in order to design an efficient graph-based classification algorithm that is described below.

\subsection{The MASC algorithm}

We propose in this section a simple, yet effective graph-based algorithm for the classification of multiple observations from the same class. Based on Proposition 1 and ignoring the constant term, we need to solve the following optimization problem:

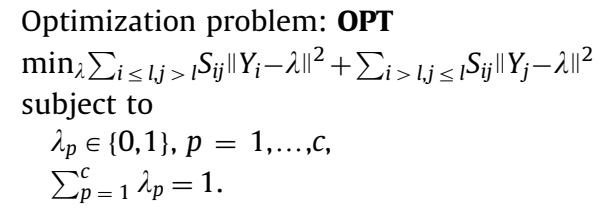

Intuitively, we seek the class that corresponds to the smoothest label assignment between labelled and unlabelled data. Observe that the above problem is a discrete optimization problem due to the constraints imposed on $\lambda$, that can be collected in a set $\Lambda$, where

$\Lambda=\left\{\lambda \in R^{c \times 1}: \lambda_{p} \in\{0,1\}, p=1, \ldots, c, \sum_{p=1}^{c} \lambda_{p}=1\right\}$.

Algorithm 1. The MASC algorithm

1: Input:

$X^{l}$ : labelled data and their labels $\left\{y_{1}, \ldots, y_{l}\right\}$

$X^{(u)}$ : multiple observations

$c$ : number of classes

$m$ : number of observations

$l$ : number of labelled data samples

2: Parameters:

$k$ : number of nearest neighbors in the graph construction

3: Output:

$\hat{p}$ : estimated unknown class.

4: Initialization:

5: $\quad$ Form the $k$-NN graph $\mathcal{G}=(\mathcal{V}, \mathcal{E})$

6: Compute the weight matrix $H \in \mathbb{R}^{n \times n}$ as in (2) and the diagonal matrix $D$, where $D_{i, i}=\sum_{j=1}^{n} H_{i j}$

7: $\quad$ Compute $S=D^{-1 / 2} H D^{-1 / 2}$.

8: for $p=1: c$ do

9:

$$
M=\left[\begin{array}{c}
Y_{l} \\
\mathbf{1} e_{p}^{\top}
\end{array}\right]
$$

10: $\quad q(p)=\sum_{i \leq l, j>l} S_{i j}\left\|M_{i}-M_{j}\right\|^{2}+\sum_{i>l, j \leq l} S_{i j}\left\|M_{i}-M_{j}\right\|^{2}$

11: end for

12: $\hat{p}=\operatorname{argmin}_{p} q(p)$

Interestingly, the search space $\Lambda$ is small. In particular, it consists of the following $c$ vectors:

$[1,0, \ldots, 0, \ldots, 0]$

$[0,1, \ldots, 0, \ldots, 0]$

$[0,0, \ldots, 1, \ldots, 0]$

$[0,0, \ldots, 0, \ldots, 1]$

Thus, one may solve OPT by enumerating all above possible solutions and pick the one $\lambda^{*}$ that minimizes $Q(\lambda)$. Then, the position of the nonzero entry in $\lambda^{*}$ yields the estimated unknown class. Notice here that the specific nature of our problem permits to avoid the use of relaxation techniques in classical label propagation [25]. Since all test samples belong to the same class, the optimal solution can be obtained with a full search, as long as the number of classes stays reasonable. We call this algorithm MAnifold-based Smoothing under Constraints (MASC) and we show its main steps in Algorithm 1.

The MASC algorithm has a computational complexity that is linear with the number of classes, and quadratic with the number of samples. The construction of the $k$-NN graph (Lines 5-7) scales as $O\left(n^{2}\right)$. Once the graph has been constructed, the enumeration of all possible solutions scales as $O(k m c)$. This is due to the fact that the matrix $S$ is sparse (i.e., $k$ nonzero entries per row) and the summands in Line 10 involve only a part of $S$. We conclude that the total computational cost of the method is $O\left(n^{2}+k m c\right)$. One may further assume that the graph among the labelled samples can be computed in an off-line step. In this case, the construction of the $k$-NN graph scales as $O(m l)$, as the Euclidean distances between each observation and the labelled set have to be computed. Overall, when the off-line cost is omitted, the total cost of the algorithm is $O(m l+k m c)$, which is linear with the 
number of classes and the number of multiple observations. This is to be contrasted, for example, with the cost of classical label propagation (see Section 3.1) that scales as $O\left(n^{3}\right)$, due to the solution of a linear system of equations, see (5).

\section{Classification of multiple images sets}

\subsection{Handwritten digit classification}

We evaluate the performance of the proposed MASC algorithm with respect to label propagation, in the context of handwritten digit classification. Multiple transformed images of the same digit class form a set of observations, which we want to assign in the correct class. We use two different data sets for our experimental evaluation: (i) a handwritten digit image collection ${ }^{1}$ and (ii) the USPS handwritten digit image collection. The first collection contains $20 \times 16$ bit binary images of "0" through " 9 ", where each class contains 39 examples. The USPS collection contains $16 \times 16$ gray-scale images of digits and each class contains 1100 examples.

Robustness to pattern transformations is a very important property of the classification of multiple observations. Transformation invariance can be reinforced into classification algorithms by augmenting the labelled examples with the so-called virtual samples, denoted hereby as $X^{(\mathrm{vs})}$ (see [13] for a similar approach). The virtual samples are essentially data samples that are generated artificially, by applying transformations to the original data samples. They are given the class labels of the original examples that they have been generated from, and are treated as labelled data. By including the virtual samples in the data set, any classification algorithm becomes more robust to transformations of the test examples. We therefore adopt this strategy in the proposed methods and we include $n^{\text {vs }}$ virtual samples $X^{(\mathrm{vs})}$ in our original data set that is finally written as $X=\left\{X^{(l)}, X^{(\mathrm{vs})}, X^{(u)}\right\}$.

Ideally, in order to obtain a k-NN graph that provides a sensible graph model of the manifold, one needs to ensure that the virtual sample set is constructed in a way such that those virtual samples that correspond to nearby transformations, are also nearby in the ambient space. However, this raises the problem of manifold discretization, which is a very challenging problem on its own and its treatment goes beyond the scope of the present work. Therefore, in this paper we use uniform discretization for the construction of the virtual sample set and assume that it is fine enough to ensure this condition.

We compare the classification performance of the MASC algorithm with the label propagation (LP) method. In LP, the estimated class is computed by majority voting on the estimated class labels computed in Eq. (5). In our experiments, we use the same $k$-NN graph in combination with the Gaussian weights from Eq. (2) in both LP and MASC methods. In order to determine the value of the parameter $\sigma$ in Eq. (2) we adopt the following process; we pick randomly 1000 examples, compute their pairwise distances and then set $\sigma$ equal to half of its median.

We first split the data sets into training and test sets by including two examples per class in the training set and the remaining are assigned to the test set. Each training sample is augmented by four virtual examples generated by successive rotations of it, where each rotation angle is sampled regularly in $\left[-40^{\circ}, 40^{\circ}\right.$. This interval has been chosen to be sufficiently small in order to avoid the confusion of digits ' 6 ' and ' 9 '. Next, in order to build the unlabelled set $X^{(u)}$ (i.e., multiple observations) of a

\footnotetext{
${ }^{1}$ http://www.cs.toronto.edu/ roweis/data.htm
}

certain class, we choose randomly a sample from the test set of this class and then we apply a random rotation on it by a random (uniformly sampled) angle $\theta \in\left[-40^{\circ}, 40^{\circ}\right]$.

The number of nearest neighbors was set to $k=5$ for both binary digit collection and the USPS data set, in both methods. These values of $k$ have been obtained by the best performance of LP on the test set. We try different sizes of the unlabelled set (i.e., multiple observations), namely $m=$ [10:20:150] (in MATLAB notation). For each value of $m$, we report the average classification error rate across 100 random realizations of $X^{(u)}$ generated from each one of the 10 classes. Thus, each point in the plot is an average over 1000 random experiments.

Figs. 4(a) and (b) show the results over the binary digits and the USPS digits image collections, respectively. Observe first that increasing the number of observations gradually improves the classification error rate of both methods. This is expected since more observations of a certain pattern give more evidence, which in turn results in higher confidence in the estimated class label. Finally, observe that the proposed MASC algorithm unsurprisingly outperforms LP in both data sets, since it is designed to exploit the particular structure of Problem 1.

a

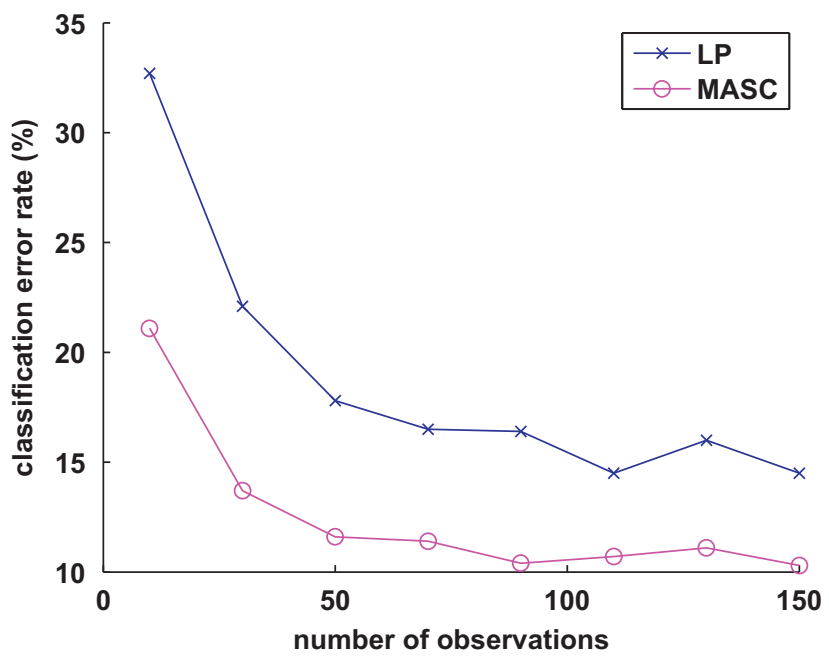

b

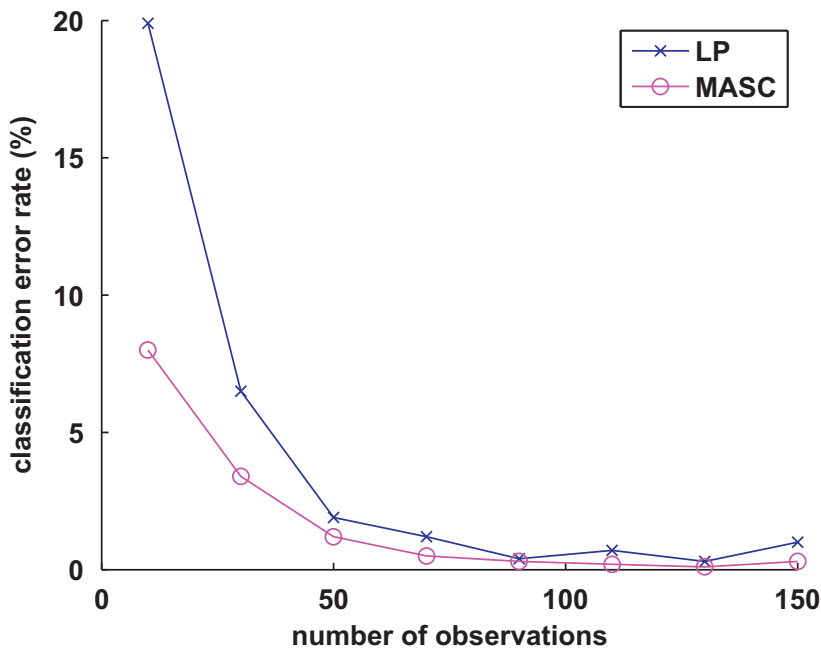

Fig. 4. Classification results measured on two different data sets. (a) Binary digits and (b) USPS digits. 


\subsection{Object recognition from multi-view image sets}

In this section we evaluate our graph-based algorithm in the context of object recognition from multi-view image sets. In this case, the different views are considered as multiple observations of the same object, and the problem is to recognize correctly this object.

The proposed MASC method implements Gaussian weights (2) and sets $k=5$ in the construction of the $k$-NN graph. We compare MASC to well-known methods from the literature, which mostly gather algorithms based on either subspace analysis or density estimation (statistical methods):

- MSM: The Mutual Subspace Method [4,21], which is the most well-known representative of the subspace analysis methods. It represents each image set by a subspace spanned by the principal components, i.e., eigenvectors of the covariance matrix. The comparison of a test image set with a training one is then achieved by computing the principal angles [5] between the two subspaces. In our experiments, the number of principal components has been set to nine, which has been found to provide the best performance.

- KMSM: MSM has been extended to its nonlinear version called the Kernel Mutual Subspace Method (KMSM) [14], in order to take into account the nonlinearity of typical image sets. The main difference of KMSM from MSM is that the images are first nonlinearly mapped into a high dimensional feature space, before modeling by linear subspaces takes place. In other words, KMSM uses kernel PCA instead of PCA in order to capture the nonlinearities in the data. In KMSM, we use the Gaussian kernel $k(x, y)=\exp \left(-\|x-y\|^{2} / 2 \sigma^{2}\right)$, where $\sigma$ is determined exactly in the same way as in the Gaussian weights of our MASC method.

- KLD: The KL-divergence algorithm by Shakhnarovich et al. [16] is the most popular representative of density-based statistical methods. It formulates the classification from multiple images as a statistical hypothesis testing problem. Under the i.i.d and the Gaussian assumptions on the image sets, the classification problem typically boils down to a computation of the KL divergence between sets, which can be computed in closed form in this case. The energy cut-off, which determines the number of principal components used in the regularization of the covariance matrices, has been set to 0.96 .

Note that the above methods can be understood as local 1-NN classification methods with different distance measures defined on image sets. In our evaluation, we use the ETH-80 image set [11], which contains 80 object classes from eight categories; apple, car, cow, cup, dog, horse, pear and tomato. Each category has 10 object classes (see Fig. 5(a)). Each object class then consists of 41 views of the object spaced evenly over the upper viewing hemisphere. Fig. 5(b) shows the 41 views from a sample car object class. We use the cropped-close128 part of the database. All provided images are of size $128 \times 128$ and they are cropped, so that they contain only the object without any border area. We downsampled the images to size $32 \times 32$ for computational ease. No further preprocessing is done.

The 41 views from each object class are split randomly into 21 training and 20 test samples. In this case, the 20 different views in the test set correspond to the multiple observations of the test object. We perform 10 random experiments where the images are randomly split into training and test sets. Table 1 presents the average object recognition rate for each method. We also report the standard deviation of each method in parentheses. Notice that the subspace methods are superior to the KLD method which a

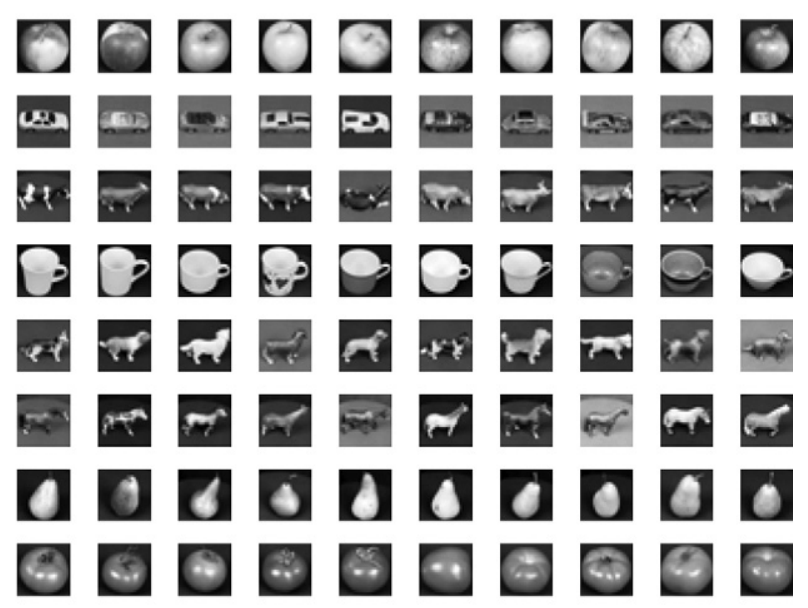

b

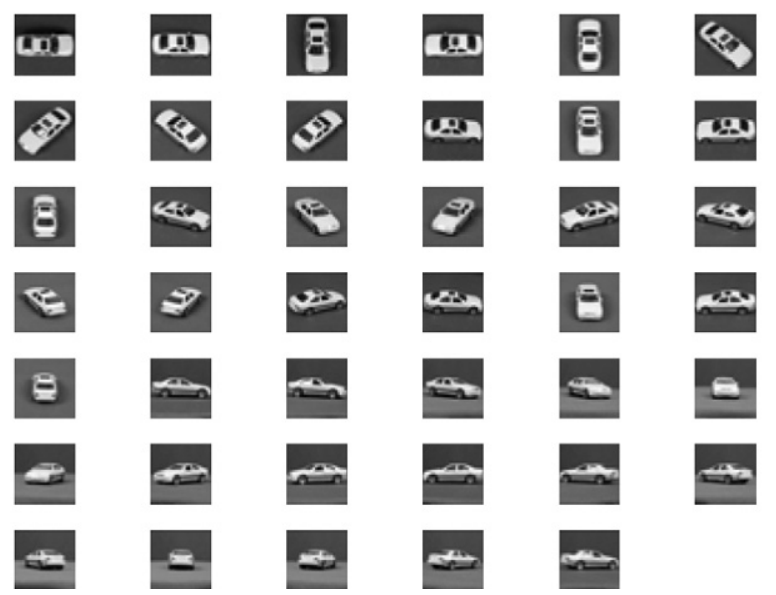

Fig. 5. Sample images from the ETH-80 database. (a) ETH- 80 and (b) 41 views of a sample car model.

Table 1

Object recognition rate in the mean (std) format, measured on the ETH-80 database.

\begin{tabular}{llll}
\hline MASC & MSM & KMSM & KLD \\
\hline $88.88(1.71)$ & $74.88(5.02)$ & $83.2500(3.4)$ & $52.5(3.95)$ \\
\hline
\end{tabular}

assumes Gaussian distribution of the data. Notice also that as one would expect, KMSM outperforms MSM that falls short of capturing the nonlinearities in the data. Finally, observe that our graph-based method clearly outperforms its competitors, as it is able to capture not only the nonlinearity, but also the manifold structure of the data.

\section{Video-based face recognition}

\subsection{Experimental setup}

In this section we evaluate our graph-based algorithm in the context of face recognition from video sequences. In this case, the different video frames are considered as multiple observations of the same person, and the problem consists in the correct classification of this person. We evaluate in this section the 
a

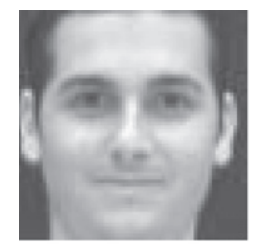

e

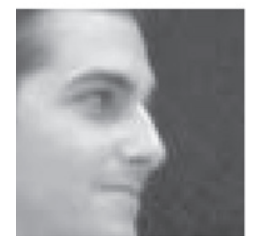

b

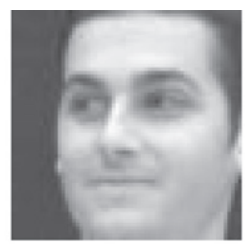

f

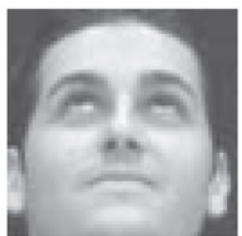

C

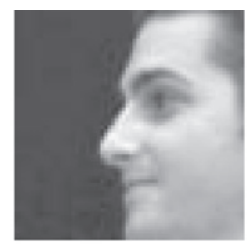

9

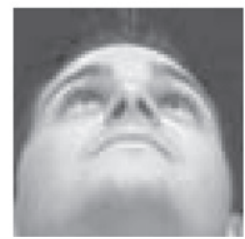

d

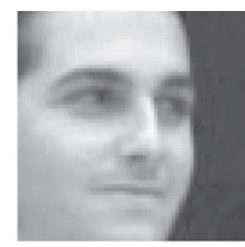

h

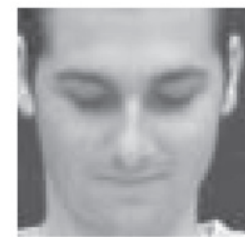

Fig. 6. Head pose variations in the VidTIMIT database. (a) pose 1 (b) pose 2 (c) pose 3 (d) pose 4 (e) pose 5 (f) pose 6 (g) pose 7 (h) pose 8.

behavior of the MASC algorithm in realistic conditions, i.e., under variations in head pose, facial expression and illumination. We compare our algorithm to state-of-the-art schemes such as KLD, MSM and KMSM, which have been described in Section 4.2. Note in passing that our algorithm does not assume any temporal order between the frames; hence, it is also applicable to the generic problem of face recognition from image sets.

We use two publicly available databases: the VidTIMIT [15] and the first subset of the Honda/UCSD [10] database. The VidTIMIT database ${ }^{2}$ contains 43 individuals and there are three face sequences obtained from three different sessions per subject. The data set has been recorded in three sessions, with a mean delay of seven days between session one and two, and six days between session two and three. In each video sequence each person performed a head rotation sequence. In particular, the sequence consists of the person moving his/her head to the left, right, back to the center, up, then down and finally return to center.

The Honda/UCSD database ${ }^{3}$ contains 59 sequences of 20 subjects. In contrast to the previous database, the individuals move their head freely, in different speed and facial expressions. In each sequence, the subjects perform free in-plane and out-ofplane head rotations. Each person has between two and five video sequences and the number of sequences per subject is variable.

For preprocessing, in both databases, we used first Viola's face detector [19] in order to automatically extract the facial region from each frame. Note that this typically results in misaligned facial images. Next, we downsampled the facial images to size $32 \times 32$ for computational ease. No further preprocessing has been performed, which brings our experimental setup closer to real testing conditions.

\subsection{Classification results on VidTIMIT}

We first study the performance of the MASC algorithm with the VidTIMIT database. Fig. 6 shows a few representative images from a sample face manifold in the VidTIMIT database. Observe the presence of large head pose variations. Fig. 7 shows the 3D projection of the manifold that is obtained using the ONPP method [9], which has been shown to be an effective tool for data visualization. Notice the four clusters corresponding to the four different head poses, i.e., looking left, right, up and down.

\footnotetext{
${ }^{2}$ http://users.rsise.anu.edu.au/ conrad/vidtimit/

${ }^{3}$ http://vision.ucsd.edu/ leekc/HondaUCSDVideoDatabase/HondaUCSD.html
}

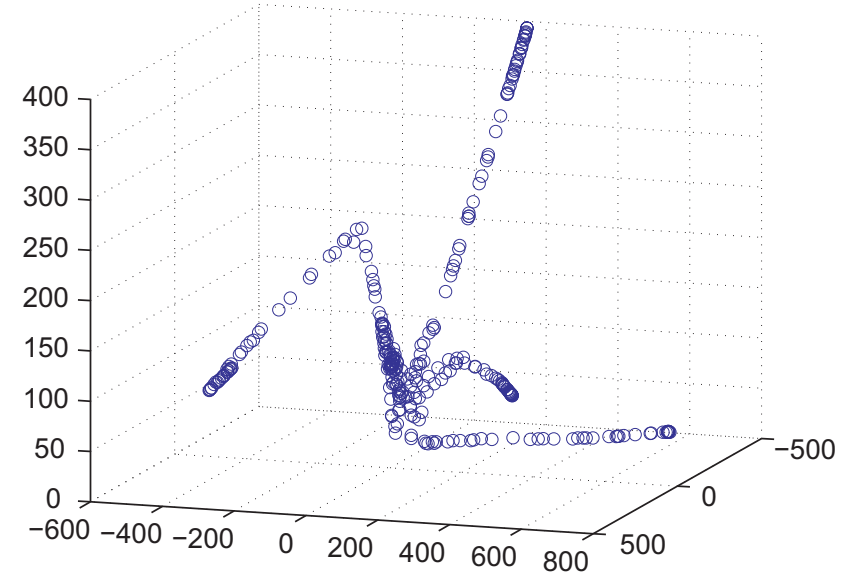

Fig. 7. A typical face manifold from the VidTIMIT database. Observe the four clusters corresponding to the four different head poses (face looking left, right, up and down)

This indicates that a graph-based method should be able to capture the geometry of the manifold and propagate class labels based on the manifold structure.

Since there are three sessions, we use the following metric for evaluating the classification performances:

$\bar{e}=\frac{1}{6} \sum_{i=1}^{3} \sum_{j=1, j \neq i}^{3} e(i, j)$,

where $e(i, j)$ is the classification error rate when the $i$ th session is used as training set and the $j$ th session is used as test set. In other words, $\bar{e}$ is the average classification error rate calculated over the following six experiments, namely $(1,2),(2,1),(1,3),(3,1),(2,3)$ and $(3,2)$.

We evaluate the video face recognition performance of all methods for diverse sizes of the training and test sets. The objective is to assess the robustness of the methods with respect to the size of the training and test set. For this reason, each image set is re-sampled as

$X_{i, r}=X_{i}(:, 1: r: n), \quad i=1, \ldots, c$.

In the above, the image set $X_{i}$ is re-sampled with step $r$, i.e., only one image for every $r$ images is kept. In our experiments, we use different values of $r$ ranging from 4 to 16 with step 4 . For each value of $r$, we measure the average classification error rate according to the relation (12). 
Table 2 presents the recognition performance, for $r$ ranging from 4 to 16 with step 4 . Fig. 8 shows graphically the same results. Observe that the KLD method that relies on density estimation is sensitive to the number of the available data. Also, notice that MSM is superior to KLD, which is expected since KLD relies on the imprecise assumption that data follow a Gaussian distribution. Furthermore, KMSM, the nonlinear variant of MSM, outperforms the latter that has trouble in capturing the nonlinear structures in the data. Finally, we observe that MASC clearly outperforms its competitors in the vast majority of cases. At the same time, it stays robust to significant re-sampling of the data, since its performance remains almost the same for each value of $r$.

Table 2

Video face recognition results on the VidTIMIT database.

\begin{tabular}{lllll}
\hline Recognition rate (\%) & MASC & MSM & KMSM & KLD \\
\hline$r=4$ & 96.51 & 91.47 & 95.74 & 84.5 \\
$r=8$ & 96.51 & 87.21 & 94.19 & 81.4 \\
$r=12$ & 94.96 & 85.66 & 92.64 & 77.52 \\
$r=16$ & 93.8 & 81.4 & 89.15 & 72.48 \\
\hline
\end{tabular}

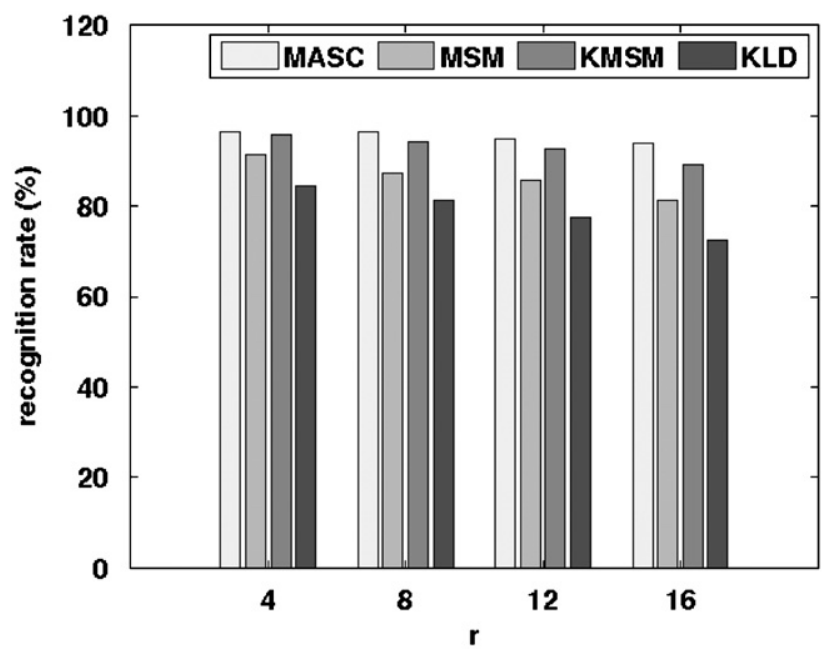

Fig. 8. Video face recognition results on the VidTIMIT database.

\subsection{Classification results on Honda/UCSD}

We further study the video-based face recognition performance on the Honda/UCSD database. Fig. 9 shows a few representative images from a sample face manifold in the Honda/UCSD database. Observe the presence of large head pose variations along with facial expressions. The projection of the manifold on the 3D space using ONPP shows again clearly the manifold structure of the data (see Fig. 10), which implies that a graph-based method is more suitable for such kind of data.

The Honda/UCSD database comes with a default splitting into training and test sets, which contains 20 training and 39 test video sequences. We use this default setup and we report the classification performance of all methods, under different data re-sampling rates. Similarly as above, both training and test image sets are re-sampled with step $r$, i.e., $X_{i, r}=X_{i}(:, 1: r: n), i=1, \ldots, c$. Table 3 presents the recognition rates, when $r$ varies from 4 to 12 with step 2. Fig. 11 shows the same results graphically. Recall that larger values of $r$ imply sparser image sets. Observe again that KLD is mostly affected by $r$, by suffering loss in performance. This is not surprising since it is a density-based method and densities cannot be accurately estimated (in general) with a few samples. MSM seems to be more robust, yielding better results than KLD, but as expected, it is inferior to KMSM in the majority of cases. Finally, MASC is again the best performer and it exhibits very high robustness against data re-sampling.

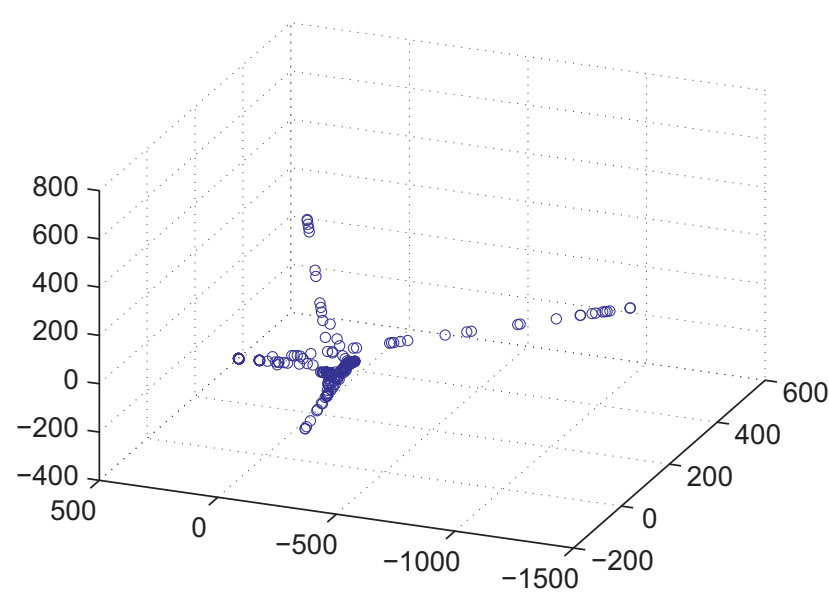

Fig. 10. A typical face manifold from the Honda/UCSD database. a

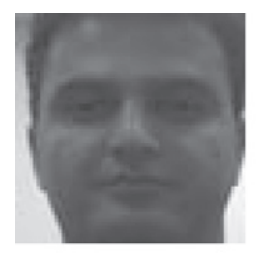

d

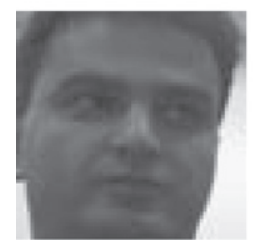

b

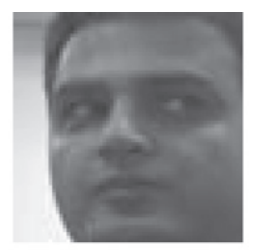

e

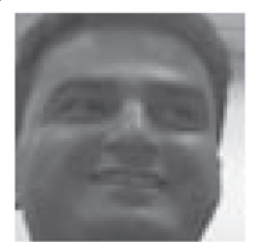

C

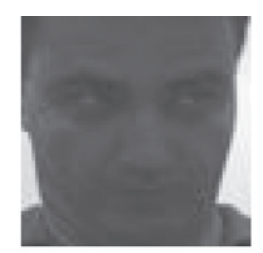

d

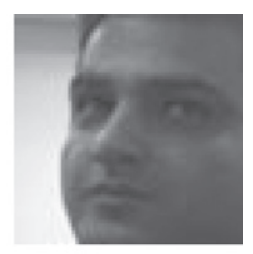

f

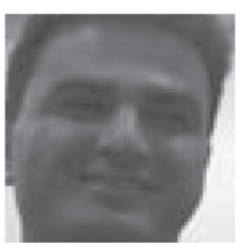

g

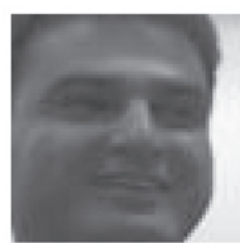

Fig. 9. Head pose variations in the Honda/UCSD database. (a) pose 1 (b) pose 2 (c) pose 3 (d) pose 4 (e) pose 5 (f) pose 6 (g) pose 7 (h) pose 8 . 
Table 3

Video face recognition results on the Honda/UCSD database.

\begin{tabular}{lcccc}
\hline Recognition rate (\%) & MASC & MSM & KMSM & KLD \\
\hline$r=4$ & 100 & 84.62 & 87.18 & 84.62 \\
$r=6$ & 100 & 84.62 & 87.18 & 79.49 \\
$r=8$ & 97.44 & 84.62 & 84.62 & 61.54 \\
$r=10$ & 97.44 & 87.18 & 84.62 & 66.67 \\
$r=12$ & 97.44 & 76.92 & 82.05 & 61.54 \\
\hline
\end{tabular}

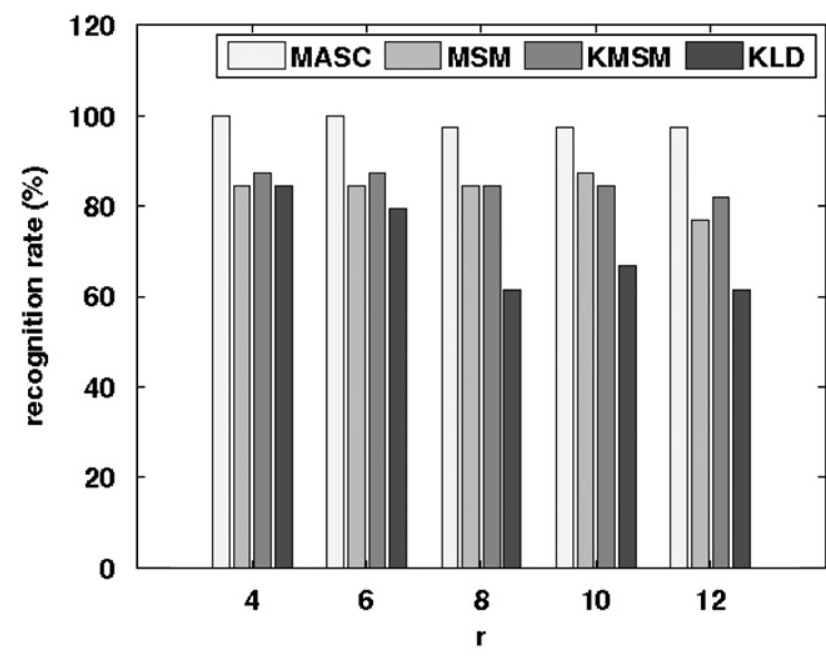

Fig. 11. Video face recognition results on the Honda/UCSD database.

Regarding the relative performance of MASC and KMSM, we should finally stress out that KMSM is a kernel technique that attempts to capture the nonlinear structure of the data by assuming a linear model after applying a nonlinear mapping of the data into a high dimensional space. Although this methodology stays generic and presents certain advantages, it is still not clear whether it is capable of capturing the individual (e.g., manifold) structure of diverse data sets. On the other hand, the MASC method explicitly relies on a graph model that may fit much better the manifold structure of the data.

If we further assume that the manifold discretization is fine enough, such that the virtual samples corresponding to close-by transformations are also close-by in the ambient space, then the proposed method can provide a way to cope with the curse of dimensionality, since the intrinsic dimension of the manifolds is typically very small.

\subsection{Video-based face recognition overview}

For the sake of completeness, we review briefly in this last section the state-of-the-art in video-based face recognition. Typically, one may distinguish between two main families of methods; those that are based on subspace analysis and those that are based on density estimation (statistical methods). The most representative methods for these two families are, respectively, the MSM [4,21] and KMSM [14] methods and the solution based on KLD [16], which have been used in the experiments above.

Among the methods based on subspace analysis, we should mention the extension of principal angles from subspaces, to nonlinear manifolds. In a recent article [20] it was proposed to represent the facial manifold by a collection of linear patches, which are recovered by a non-iterative algorithm that augments the current patch until the linearity criterion is violated. This manifold representation allows for defining the distance between manifolds as integration of distances between linear patches. For comparing two linear patches, the authors propose a distance measure that is a mixture between (i) the principal angles and (ii) exemplar-based distance. However, it is not clearly justified why such a mixture is needed and what is the relative benefit over the individual distances. Moreover, their proposed method requires the computation of both geodesic and Euclidean distances as well as setting four parameters. On the contrary, our MASC method needs only one parameter $(k)$ to be set and it requires the computation of the Euclidean distances only. Note finally that their method achieves comparable results with MASC on the Honda/UCSD database, but at a higher computational cost and at the price of tuning four parameters.

Along the same lines, the authors in [7] propose a similarity measure between manifolds that is a mixture of similarity between subspaces and similarity between local linear patches. Each individual similarity is based on a weighted combination of principal angles and those weights are learned by AdaBoost for improved discriminative performance. In contrast to the previous paper [20], the linear patches are extracted here using mixtures of Probabilistic PCA (PPCA). PPCA mixture fitting is a highly nontrivial task, which requires an estimate of the local principal subspace dimension and it also involves model selection. This step is quite computationally intensive, as noted in [20].

The main limitation of the statistical methods such as KLD [16] is the inadequacy of the Gaussianity assumption of face images sets; face sequences rather have a manifold structure. The test video frames are moreover not independent, so that the i.i.d assumption is unrealistic as well. The authors in [1] therefore extend the work of $\mathrm{KL}$ divergence by replacing the Gaussian densities by Gaussian Mixture Models (GMMs), which provides a more flexible method for density estimation. However, the KL divergence in this case cannot be computed in a closed form, which makes the authors to resort to Monte Carlo simulations that are quite computationally intensive.

Finally, there have been a few other methods that cannot be directly categorized in the above families of methods. The authors in [23] propose ensemble similarity metrics that are based on probabilistic distance measures, evaluated in reproducing Kernel Hilbert spaces. All computations are performed under the Gaussianity assumption, which is unfortunately not realistic for facial manifolds.

In [24], the authors provide a probabilistic framework for face recognition from image sets. They model the identity as a discrete or continuous random variable and they provide a statistical framework for estimating the identity by marginalizing over face localization, illumination and head pose. Illumination-invariant basis vectors are learned for each (discretized) pose and the resulting subspace is used for representing the low-dimensional vector that encodes the subject identity. However, the statistical framework requires the computation of several integrals that are numerically approximated. Also, the proposed method assumes that training images are available for every subject at each possible pose and illumination, which is hard to satisfy in practice.

Liu and Chen in [12] proposed a methodology based on adaptive hidden Markov models for video-based face recognition. The temporal dynamics of each subject are learned during training and subsequently used for recognition. However, the proposed approach assumes temporal order of the frames in the face sequence and unfortunately it is not applicable to the more generic problem of recognition from image sets. The study in [6] further investigates how the performance of the above approach is affected by the face sequence length and the image quality. 


\section{Conclusions}

In this paper we have addressed the problem of classification of multiple observations of the same object. We have proposed to exploit the specific structure of this problem in a graph-based algorithm inspired by label propagation. The graph-based algorithm relies on the manifold structure in order to estimate the unknown class, under the constraint that all observations correspond to the same class. We have formulated this process as a discrete optimization problem that can be solved efficiently by a low complexity algorithm.

We provide experimental results that illustrate the performance of the proposed solution for the classification of handwritten digits, for object recognition and for video-based face recognition. In the two latter cases, the graph-based solution outperforms state-ofthe-art methods on three publicly available data sets. If we also take into account the fact that the method is simple, efficient, and does not make use of specialized features (at least in its current form), it clearly suggests that graph methods are certainly very promising and have a great potential in this field. In our future work, we plan to extend the method with specialized features that are tuned to the application at hand.

\section{References}

[1] O. Arandjelović, G. Shakhnarovich, J. Fisher, R. Cipolla, T. Darrell, Face recognition with image sets using manifold density divergence, IEEE International Conference on Computer Vision and Pattern Recognition (CVPR), vol. 1, 2005, pp. 581-588.

[2] F. Bach, M. Jordan, Learning spectral clustering, in: Advances in Neural Information Processing Systems 16 (NIPS), 2003.

[3] O. Chapelle, B. Scholkopf, A. Zien, Semi-Supervised Learning, MIT Press, 2006.

[4] K. Fukui, O. Yamaguchi, Face recognition using multi-viewpoint patterns for robot vision, in: International Symposium on Robotics Research, vol. 15, 2005, pp. 192-201.

[5] G.H. Golub, C.V. Loan, Matrix Computations, third ed., The John Hopkins University Press, Baltimore, 1996.

[6] A. Hadid, M. Pietikainen, From still image to video-based face recognition: an experimental analysis, in: Sixth IEEE International Conference on Automatic Face and Gesture Recognition, 2004, pp. 813-818.

[7] T.-K. Kim, O. Arandjelovic, R. Cipolla, Boosted manifold principal angles for image set-based recognition, Pattern Recognition 40 (2007) 2475-2484.

[8] E. Kokiopoulou, S. Pirillos, P. Frossard, Graph-based classification for multiple observations of transformed patterns, in: IEEE International Conference on Pattern Recognition (ICPR), December 2008, pp. 20-24.
[9] E. Kokiopoulou, Y. Saad, Orthogonal neighborhood preserving projections: a projection-based dimensionality reduction technique, IEEE Transactions on Pattern Analysis and Machine Intelligence 29 (12) (2007) 2143-2156.

[10] K.C. Lee, J. Ho, M.H. Yang, D. Kriegman, Video-based face recognition using probabilistic appearance manifolds, in: IEEE International Conference on Computer Vision and Pattern Recognition (CVPR), 2003, pp. 313-320.

[11] B. Leibe, B. Schiele, Analyzing appearance and contour based methods for object categorization, in: International Conference on Computer Vision and Pattern Recognition (CVPR'03) Madison, Wisconsin, 2003.

[12] X. Liu, T. Chen, Video-based face recognition using adaptive hidden markov models, IEEE International Conference on Computer Vision and Pattern Recognition (CVPR), vol. 1, 2003, pp. I-340-I-345.

[13] A. Pozdnoukhov, S. Bengio, Graph-based transformation manifolds for invariant pattern recognition with kernel methods, in: IEEE International Conference on Pattern Recognition (ICPR), 2006.

[14] H. Sakano, N. Mukawa, Kernel mutual subspace method for robust facial image recognition, Fourth International Conference on Knowledge-based Intelligent Engineering Systems and Allied Technologies (KES 2000), vol. 1, 2000, pp. 245-248.

[15] C. Sanderson, Biometric Person Recognition: Face, Speech and Fusion, VDMVerlag, 2008.

[16] G. Shakhnarovich, J.W. Fisher, T. Darrel, Face recognition from long-term observations, European Conference on Computer Vision (ECCV), vol. 3, 2002, pp. 851-868.

[17] C. Stauffer, Minimally-supervised classification using multiple observation sets, in: IEEE International Conference on Computer Vision (ICCV), 2003.

[18] M. Szummer, T. Jaakkola, Partially labeled classification with markov random walks, in: Advances in Neural Information Processing Systems (NIPS), 2002.

[19] P. Viola, M. Jones, Robust real-time face detection, International Journal of Computer Vision 57 (2) (2004) 137-154.

[20] R. Wang, S. Shan, S. Chen, W. Gao, Manifold-manifold distance with application to face recognition based on image set, in: IEEE International Conference on Computer Vision and Pattern Recognition (CVPR), 2008

[21] O. Yamaguchi, K. Fukui, K. Maeda, Face recognition using temporal image sequence, in: IEEE International Conference on Automatic Face and Gesture Recognition, 1998, pp. 318-323.

[22] D. Zhou, O. Bousquet, T.N. Lal, J. Weston, B. Schölkopf, Learning with local and global consistency, in: Advances in Neural Information Processing Systems (NIPS), 2003.

[23] S. Zhou, R. Chellappa, From sample similarity to ensemble similarity: probabilistic distance measures in reproducing kernel hilbert space, IEEE Transactions on Pattern Analysis and Machine Intelligence 28 (6) (2006) 917-929.

[24] S.K. Zhou, R. Chellappa, Probabilistic identity characterization for face recognition, IEEE International Conference on Computer Vision and Pattern Recognition (CVPR), vol. 2, 2004, pp. 805-812.

[25] X. Zhu, Z. Ghahramani, Learning from labeled and unlabeled data with Label Propagation, Technical Report CMU-CALD-02-107, Carnegie Mellon University, Pittsburgh, 2002.

[26] X. Zhu, Z. Ghahramani, J. Lafferty, Semi-supervised learning using Gaussian fields and harmonic functions, in: 20th International Conference on Machine Learning (ICML), 2003.

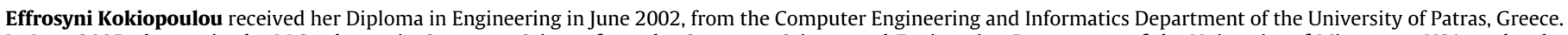

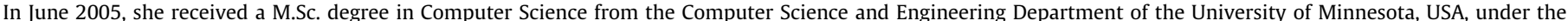

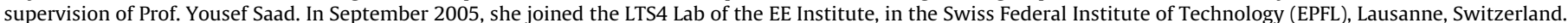

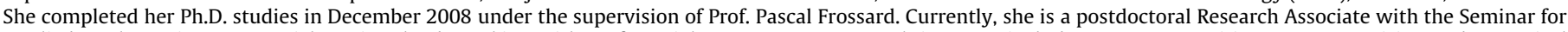

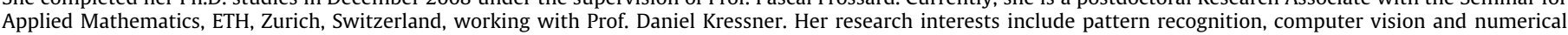
linear algebra.

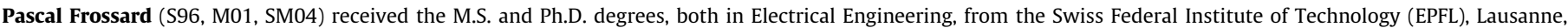

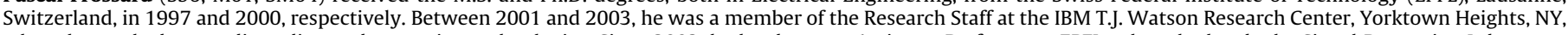

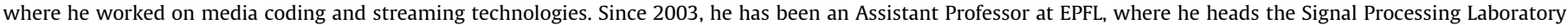

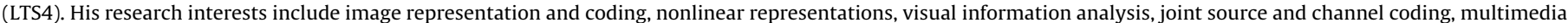

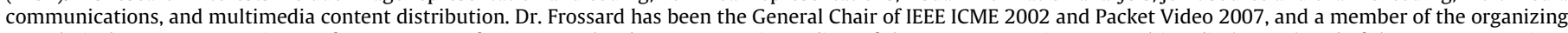

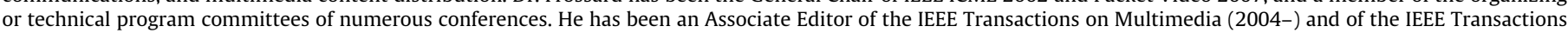

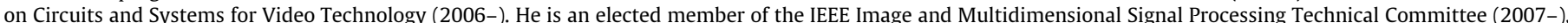

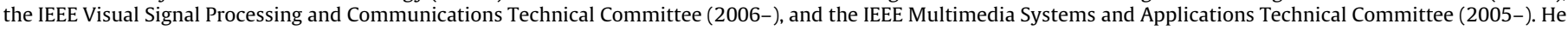

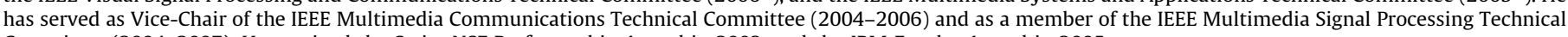
Committee (2004-2007). He received the Swiss NSF Professorship Award in 2003, and the IBM Faculty Award in 2005. 